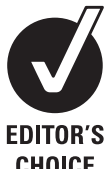

CHOICE
- Supplementary data are published online only. To view these files please visit the journal online (http://thorax.bmj. com)

${ }^{1}$ Arizona Respiratory Center, University of Arizona, Tucson, Arizona, USA

${ }^{2}$ Centre for Research in Environmental Epidemiology (CREAL), IMIM-Hospital del Mar, CIBERESP, Barcelona, Spain

${ }^{3} \mathrm{Mel}$ and Enid Zuckerman College of Public Health University of Arizona, Arizona, USA

${ }^{4}$ Department of Pharmacology, University of Arizona, Tucson, Arizona, USA

\section{Correspondence to}

Stefano Guerra, Arizona

Respiratory Center, University of Arizona, 1501 N. Campbell Ave, P 0 Box 245030, Tucson, AZ 85724-5030, USA;

sguerra@arc.arizona.edu

Received 25 August 2009 Accepted 13 March 2010

\title{
Morbidity and mortality associated with the restrictive spirometric pattern: a longitudinal study
}

\author{
Stefano Guerra, ${ }^{1,2}$ Duane L Sherrill, ${ }^{1,3}$ Claire Venker, ${ }^{1,3}$ Christina M Ceccato, ${ }^{1}$ \\ Marilyn Halonen, ${ }^{1,4}$ Fernando D Martinez ${ }^{1}$
}

\section{ABSTRACT \\ Background Recent studies have suggested that} a restrictive pattern assessed with a single spirometric test is associated with increased morbidity and mortality. This study was undertaken to determine demographic, clinical and mortality profiles of subjects with either a recurrent or an inconsistent restrictive spirometric pattern assessed prospectively.

Methods Data from 2048 adult participants in the population-based TESAOD study were analysed. Normal (forced expiratory volume in $1 \mathrm{~s} /$ forced vital capacity (FEV $1 / F V C$ ) ratio $\geq 70 \%$ and $\mathrm{FVC} \geq 80 \%$ predicted), restrictive $\left(\mathrm{FEV}_{1} / \mathrm{FVC} \geq 70 \%\right.$ and $\mathrm{FVC}<80 \%$ predicted) and obstructive ( $\left.\mathrm{FEV}_{1} / \mathrm{FVC}<70 \%\right)$ patterns were assessed at the enrolment survey in 1972 and in 11 subsequent follow-up surveys up to 1996. Demographic and clinical characteristics were measured at enrolment and vital status and cause of death were assessed at January 2005.

Results Overall, $12 \%$ of participants had a restrictive spirometric pattern at enrolment. They were less likely to be male, to smoke and to have asthma, and had lower $\lg \mathrm{E}$ levels than subjects in the obstructive group. Among subjects with a restrictive pattern at enrolment, 38\% developed an obstructive pattern during follow-up. The remaining $62 \%$ had either a recurrent (restrictive pattern $\geq 50 \%$ of follow-up surveys) or inconsistent (restrictive pattern $<50 \%$ of follow-up surveys) longitudinal restrictive pattern. The recurrent and inconsistent restrictive groups had increased mortality risk for allcause (adjusted HR 1.7 (95\% Cl 1.3 to 2.3) and 1.9 (95\% $\mathrm{Cl} 1.4$ to 2.6$)$, respectively), heart disease $(2.0(95 \% \mathrm{Cl}$ 1.3 to 3.1$)$ and $2.7(95 \% \mathrm{Cl} 1.7$ to 4.3$))$, stroke (2.4 (95\% $\mathrm{Cl} 0.9$ to 6.3$)$ and $3.5(95 \% \mathrm{Cl} 1.2$ to 9.8$))$ and diabetes (8.0 (95\% Cl 2.9 to 21.8) and 6.0 (95\% Cl 1.9 to 19.2)).

Conclusions The restrictive spirometric pattern identifies a pulmonary condition that is distinguishable from obstructive lung disease and is associated with an increased risk of life-threatening comorbidities.

\section{INTRODUCTION}

A reduction in the forced expiratory volume in $1 \mathrm{~s}$ $\left(\mathrm{FEV}_{1}\right)$ has been associated with respiratory symptoms, functional limitation and mortality risk. ${ }^{1} 2$ These findings are partly explained by morbidity and mortality associated with obstructive lung diseases, particularly chronic obstructive pulmonary disease (COPD). COPD, which is defined functionally by a post-bronchodilator ratio between $\mathrm{FEV}_{1}$ and forced vital capacity (FVC) $<70 \%{ }^{3}$ is one of the leading causes of mortality and quality-adjusted life years lost in the USA as well as worldwide. ${ }^{4}$
At the population level, however, a significant proportion of subjects with reduced $\mathrm{FEV}_{1}$ levels also have reduced FVC and a normal $\mathrm{FEV}_{1} / \mathrm{FVC}$ ratio. Thus, COPD is not the only explanation for the excess mortality in individuals with low $\mathrm{FEV}_{1}$. Indeed, a strong link between low FVC and mortality risk has long been known, ${ }^{6}$ and recent evidence suggests that a restrictive spirometric pattern is relatively common and accounts for an important portion of morbidity and mortality related to lung function impairment. Epidemiolog ical studies have found that, in the general population, between $7 \%$ and $13 \%$ of adults have FVC values $<80 \%$ of expected for their sex, age and height in the presence of an $\mathrm{FEV}_{1} / \mathrm{FVC}$ ratio $\geq 70 \%,{ }^{7-10}$ and that these individuals are at increased risk for all-cause and cardiovascular mortality. ${ }^{8} 9$ This restrictive spirometric pattern is also associated with significant functional impairment $^{7}$ and various comorbid conditions, including diabetes, ${ }^{11} 12$ metabolic syndrome, ${ }^{13}{ }^{14}$ hypertension, $^{12} 15$ stroke $^{15}$ and cardiovascular disease. ${ }^{16}$ Although some of the above studies assessed the relation of lung function to morbidity and mortality prospectively, they used a single spirometric test at enrolment to measure lung function.

Low FVC values assessed by spirometry can also be found in a proportion of normal subjects who complete spirometry using submaximal inspiratory efforts and in subjects with air trapping associated with obstructive lung diseases such as asthma and COPD. Although assessment of total lung capacity by plethysmography is the only conclusive test to differentiate truly restricted subjects from misclassified obstructed and normal individuals, this test-unlike spirometry-is time-consuming, costly and needs to be completed in a specialised setting, precluding its potential use in screening programmes on large populations. It is reasonable to hypothesise that a longitudinal follow-up based on serial spirometric tests on the same subjects, as opposed to a single spirometric assessment, may help distinguish cases with a true restrictive spirometric pattern from those in which the restrictive pattern is a manifestation of an underlying airway obstruction. However, no previous study has attempted to characterise prospectively the restrictive spirometric pattern and to evaluate the specific morbidity and mortality burden associated with such longitudinal patterns.

In a large population-based prospective cohort we sought to determine demographic, clinical and mortality profiles associated with recurrent and inconsistent restrictive longitudinal patterns and to compare them to those of obstructive longitudinal patterns. 


\section{METHODS \\ Study population}

The Tucson Epidemiological Study of Airway Obstructive Disease (TESAOD) is a population-based prospective cohort study initiated in Tucson, Arizona, USA in 1972. Details of the enrolment process have been previously reported. ${ }^{17}$ Briefly, using a multistage stratified cluster sample of Anglo-white Tucson households (based on the 1970 census block statistics), 2989 households were originally approached, of which 559 (18.7\%) refused to participate, 350 (11.7\%) were ineligible, 329 (11\%) were vacant dwelling units and 96 (3.2\%) were classified as permanently not at home. A total of 1655 households finally participated in the enrolment survey (survey 1, completed in 1972-3). At enrolment, 2754 white participants (age range 6-95 years) from these households completed both a standardised respiratory questionnaire and spirometric lung function tests with a pneumotachygraphic device according to methods previously described. ${ }^{18}$ Twelve additional follow-up surveys were completed approximately every 2 years up to 1996, in which participants completed the same standardised questionnaire and (with the only exception of survey 4) spirometric lung function tests. To reduce the loss to follow-up of participants who moved out of Tucson during the study, the study nurses travelled to various states in the USA to perform lung function tests and collect questionnaire data from many of these participants.

For the present study, we used data from 2048 participants who were aged 21-80 years, did not report having ever had lung/chest surgery, were not pregnant and completed acceptable lung function tests at enrolment.

\section{Baseline and longitudinal spirometric patterns and covariates}

Baseline spirometric patterns at enrolment were defined as normal ( $\mathrm{FEV}_{1} / \mathrm{FVC} \geq 70 \%$ and $\mathrm{FVC} \geq 80 \%$ predicted), restrictive $\left(\mathrm{FEV}_{1} / \mathrm{FVC} \geq 70 \%\right.$ and $\mathrm{FVC}<80 \%$ predicted) or obstructive $\left(\mathrm{FEV}_{1} / \mathrm{FVC}<70 \%\right.$ independent of FVC values) using reference equations generated in the same population by Knudson and colleagues. ${ }^{19}$

In addition, among subjects who had at least one follow-up survey, the following six longitudinal groups were generated based on the combination of spirometric patterns at enrolment and follow-up surveys:

- Consistent normal (normal pattern at enrolment and in all completed follow-up surveys).

- Recurrent restrictive (restrictive pattern at enrolment and in at least $50 \%$ of the follow-up surveys, plus never obstructive pattern).

- Inconsistent restrictive (restrictive pattern at enrolment and in $<50 \%$ of the follow-up surveys, plus never obstructive pattern).

- Recurrent obstructive (obstructive pattern at enrolment and in at least $50 \%$ of the follow-up surveys, plus never restrictive pattern).

- Inconsistent obstructive (obstructive pattern at enrolment and in $<50 \%$ of the follow-up surveys, plus never restrictive pattern).

- Mixed restrictive/obstructive (restrictive pattern at enrolment and obstructive pattern in at least one of the follow-up surveys; or obstructive pattern at enrolment and restrictive pattern in at least one of the follow-up surveys).

Two additional longitudinal spirometric patterns (Incident restrictive: normal pattern at enrolment and development of a restrictive pattern during the follow-up; and Incident obstructive: normal pattern at enrolment and development of an obstructive pattern during the follow-up) were also identified but, because of the specific goals of the present study, they were not included in the main analyses. The results from these two incident groups are presented in the online supplement.

At enrolment, in addition to an in-depth standardised respiratory questionnaire and lung function tests, height and weight were measured by research nurses and skin prick tests and blood eosinophil counts performed (see online supplement). Obesity was defined as a body mass index $(\mathrm{BMI}) \geq 30 \mathrm{~kg} / \mathrm{m}^{2}$ and being overweight as a BMI $\geq 25$ and $<30 \mathrm{~kg} / \mathrm{m}^{2}$. Positive skin prick tests were defined as a weal $\geq 2 \mathrm{~mm}$ larger than the control weal for at least one of five tested allergens and eosinophilia as eosinophils $>4 \%$. Measurement of total serum IgE was also completed using the paper radioimmunosorbent test (PRIST; Pharmacia Diagnostics, Piscataway, New Jersey, USA) method. ${ }^{20}$

\section{Assessment of mortality}

Vital status of TESAOD participants at January 2005 was determined by direct contact with the family or designated next of kin of the participant, linkage with the Social Security Death Index ${ }^{21}$ and-for deaths that occurred after 1978-linkage with the National Death Index (NDI). ${ }^{22}$ Underlying causes of death were obtained from NDI records for deaths that occurred after 1978 and directly from death certificates for deaths that occurred earlier (see table E1 in online supplement).

\section{Statistical analyses}

Because spirometric patterns differed substantially by age, demographic and clinical characteristics were compared across different spirometric patterns using linear and logistic regression models adjusted by age. IgE values were log-transformed to achieve normalisation.

The relation of longitudinal spirometric patterns to all-cause and cause-specific mortality was investigated in sex-, age- and BMI-adjusted Cox proportional hazards models. Time to event was defined as the time between enrolment and the date of death for deceased participants and as the time between enrolment and January 2005 for alive subjects. In analyses for specific causes of death, cases were represented by participants who had that specific disease as the underlying cause of death. However, because only 10 subjects had diabetes identified as the underlying cause of death, in Cox models for diabetes mortality events were defined as cases with diabetes indicated as either underlying cause of death or co-existing condition on the death certificate.

\section{RESULTS}

\section{Comparison of participants included and excluded from the present study}

At the enrolment survey, 2408 TESAOD participants were aged 21-80 years and did not report being pregnant or having ever had lung or chest surgery. Of these, 2048 (85\%) completed lung function tests and were included in the present study. Participants included in the present study did not differ from those excluded in terms of age, sex, BMI, education, smoking or skin test positivity. The only significant difference between the two groups was that subjects included in the present study were more likely to have physician-confirmed asthma than subjects excluded from the study $(11.1 \%$ vs $7.5 \%$, respectively; $p=0.04)$.

Demographic and clinical characteristics at enrolment (1972-3) At enrolment, of the 2048 subjects included in the present study, $1505(74 \%)$ had normal lung function, 249 (12\%) had a spirometric restrictive pattern and 294 (14\%) had an obstructive 
pattern. The three groups differed substantially by age distribution $(p<0.001)$. In order to reduce the impact of age differences across the three groups, demographic and clinical characteristics are compared in table 1 after adjustment for age. The restrictive pattern was associated with obesity and asthma. However, fewer than $14 \%$ of subjects with the restrictive pattern were obese and no differences in the proportion of overweight subjects were found among the restrictive $(35 \%)$, normal (34\%) and obstructive (34\%) pattern. Overall, 14\% of subjects with the restrictive pattern had a physician-confirmed diagnosis of asthma compared with $8 \%$ of subjects in the normal pattern. However, these two patterns did not differ in terms of skin prick tests, eosinophilia or IgE levels. In contrast, subjects with the obstructive pattern were more likely to be males and smokers and to have asthma, eosinophilia and elevated IgE than subjects with normal lung function. Overall, $70 \%$ of subjects with the obstructive pattern were heavy smokers ( $\geq 20$ packyears) and/or had asthma compared with $42 \%$ of subjects with the restrictive pattern and $30 \%$ of subjects with normal lung function.

Both the restrictive and the obstructive pattern were associated with chronic bronchitis, shortness of breath with wheezing, dyspnoea and a positive history of pneumonia compared with the normal pattern (table 1), although the majority of these associations were stronger among subjects with the obstructive pattern than in those with the restrictive pattern. The latter group, however, tended to have higher rates of heart disease, stroke and diabetes.

\section{Longitudinal spirometric patterns (1972-96)}

Among the 2048 subjects included in this study, 24 (1\%) died before the first follow-up survey $(10$ subjects in the normal group, 5 in the restrictive pattern group and 9 in the obstructive spirometric pattern group). Among the remaining 2024 subjects,
1798 (89\%) had follow-up data, with an average of 12.2 years of follow-up and an average of 6.3 lung function tests completed during the study. Of these, 126 subjects had an incident restrictive pattern and 304 had an incident obstructive pattern. The findings on these groups are presented in tables E2 and E3 in the online supplement. The remaining 1368 participants were divided into the six longitudinal spirometric patterns shown in table 2. Of note, among the 211 subjects with spirometric restriction at enrolment and available follow-up data, 69 (33\%) had a recurrent restrictive pattern, 62 (29\%) an inconsistent restrictive pattern and 80 (38\%) developed airflow limitation during the study follow-up and accounted for the majority of subjects included in the mixed restrictive/obstructive pattern group.

The demographic and clinical characteristics at enrolment of the subjects in the six longitudinal spirometric patterns are shown in table 2. Compared with the recurrent obstructive group, subjects in the recurrent restrictive group appeared more likely to be female and obese and less likely to smoke and to have asthma, eosinophilia and elevated IgE. The characteristics of subjects with the recurrent and inconsistent restrictive patterns were very similar, with the former differing from the latter only in terms of older age and lower FVC percentage predicted at baseline ( $68 \%$ vs $75 \%, p<0.001$; data not shown).

\section{All-cause and cause-specific mortality associated with longitudinal patterns (1972-2005)}

After adjusting for sex, age and BMI, subjects with the recurrent restrictive pattern had a $70 \%$ increased mortality risk (range $30-130 \%$ ) and subjects with the inconsistent restrictive pattern had a $90 \%$ increased mortality risk (range 40-160\%) compared with subjects who had consistently normal lung function during the study follow-up (table 3). Among the corresponding obstructive longitudinal patterns, only the recurrent obstructive

Table 1 Demographic and clinical characteristics at enrolment of TESAOD participants according to their lung function at enrolment and vital status at January 2005

\begin{tabular}{|c|c|c|c|c|}
\hline & \multicolumn{3}{|c|}{ Lung function at enrolment } & \multirow[b]{2}{*}{ p Value* } \\
\hline & Normal $(N=1505)$ & $\begin{array}{l}\text { Restrictive spirometric } \\
\text { pattern }(N=249)\end{array}$ & $\begin{array}{l}\text { Obstructive spirometric } \\
\text { pattern }(N=294)\end{array}$ & \\
\hline Mean $\pm S D$ age (years) & $46 \pm 17$ & $60 \pm 15$ & $60 \pm 15$ & NA \\
\hline Sex: \% female & 56.3 & 67.1 & 46.9†, $\ddagger$ & $<0.001$ \\
\hline Obesity: $\%$ with $\mathrm{BMI} \geq 30 \mathrm{~kg} / \mathrm{m}^{2}(\mathrm{n}=1978)$ & 6.8 & $13.6 \dagger$ & $6.4 \ddagger$ & 0.004 \\
\hline Years of formal education: $\%$ with $>12$ years & 45.8 & 37.8 & 32.0 & 0.27 \\
\hline Ever smoking: $\%$ with $\geq 1$ pack-year $(n=2046)$ & 53.9 & 50.6 & $77.1 \dagger, \ddagger$ & $<0.001$ \\
\hline Pack-years: geometric mean among ever smokers $(n=1163)$ & 14.1 & 22.5 & $28.9 \dagger$ & 0.006 \\
\hline Physician-confirmed asthma: \% positive $(\mathrm{n}=2046)$ & 8.2 & $14.1+$ & $23.5 \dagger, \ddagger$ & $<0.001$ \\
\hline Allergy skin tests: \% positive $(n=2012)$ & 39.7 & 25.9 & 31.6 & 0.28 \\
\hline Eosinophilia: \% positive $(n=1542)$ & 7.4 & 9.6 & $12.8 \dagger$ & 0.01 \\
\hline Total serum IgE in IU/ml: geometric mean ( $n=1836)$ & 27.5 & 21.5 & $37.8 \dagger, \ddagger$ & $<0.001$ \\
\hline Chronic cough and phlegm: \% positive & 7.7 & $13.7 \dagger$ & $31.0 \dagger, \ddagger$ & $<0.001$ \\
\hline Shortness of breath with wheezing: \% positive $(n=2043)$ & 16.9 & $27.7 \dagger$ & 42.8†, ‡ & $<0.001$ \\
\hline $\begin{array}{l}\geq \text { Grade } 2 \text { on the modified MRC dyspnoea scale } †: \% \text { positive } \\
(\mathrm{n}=2038)\end{array}$ & 9.5 & $24.0 \dagger$ & $34.9 \dagger, \neq$ & $<0.001$ \\
\hline Ever pneumonia: \% positive & 23.0 & $40.6 \dagger$ & $39.5 \dagger$ & $<0.001$ \\
\hline Ever 'heart trouble': \% positive & 13.8 & 26.1 & 20.7 & 0.36 \\
\hline Ever stroke: \% positive & 1.1 & $6.0 \dagger$ & 3.1 & 0.05 \\
\hline Diabetes: \% positive & 3.0 & 7.2 & 4.4 & 0.29 \\
\hline Deceased at January 2005: \% deceased & 46.3 & 82.7 & 84.0 & NA \\
\hline
\end{tabular}

$\mathrm{N}=2048$ unless otherwise specified.

Statistical comparisons are completed across the three groups after adjustment for age ( $p$ value reported in the last column) as well as between pairs after adjustment for age and Bonferroni correction.

*p Value for the comparison across the three groups after adjustment for age.

†Significantly different from normal after adjustment for age and Bonferroni correction.

$\ddagger$ Significantly different from restrictive spirometric pattern after adjustment for age and Bonferroni correction. 
Table 2 Demographic and clinical characteristics at enrolment and length of follow-up of subjects included in the six longitudinal spirometric patterns

\begin{tabular}{|c|c|c|c|c|c|c|c|}
\hline & \multicolumn{6}{|c|}{ Longitudinal spirometric patterns } & \multirow[b]{2}{*}{$\begin{array}{l}\mathbf{p} \\
\text { Value* }\end{array}$} \\
\hline & $\begin{array}{l}\text { Consistent } \\
\text { normal } \\
(\mathrm{N}=913)\end{array}$ & $\begin{array}{l}\text { Recurrent } \\
\text { restrictive } \\
(\mathrm{N}=69)\end{array}$ & $\begin{array}{l}\text { Inconsistent } \\
\text { restrictive } \\
(\mathrm{N}=62)\end{array}$ & $\begin{array}{l}\text { Recurrent } \\
\text { obstructive } \\
\text { (N=155) }\end{array}$ & $\begin{array}{l}\text { Inconsistent } \\
\text { obstructive } \\
\text { (N=54) }\end{array}$ & $\begin{array}{l}\text { Mixed restrictive/ } \\
\text { obstructive } \\
(\mathrm{N}=115)\end{array}$ & \\
\hline Sex: \% female & 59.1 & 76.8 & 69.4 & 38.7 & 59.3 & 67.0 & $<0.001$ \\
\hline Obesity: $\%$ with $\mathrm{BMI} \geq 30 \mathrm{~kg} / \mathrm{m}^{2}(\mathrm{n}=1325)$ & 6.3 & 15.4 & 16.7 & 3.3 & 9.6 & 12.5 & 0.006 \\
\hline $\begin{array}{l}\text { Ever smoking: } \% \text { with } \geq 1 \\
\text { pack-year }(n=1367)\end{array}$ & 48.4 & 37.7 & 50.0 & 82.6 & 73.6 & 58.3 & $<0.001$ \\
\hline Eosinophilia: \% positive $(n=1035)$ & 7.1 & 6.3 & 10.9 & 14.0 & 14.0 & 13.8 & 0.05 \\
\hline $\begin{array}{l}\text { Total serum lgE in IU/ml: geometric mean } \\
(n=1221)\end{array}$ & 25.2 & 19.3 & 29.1 & 52.2 & 25.7 & 23.3 & $<0.001$ \\
\hline Mean \pm SD total follow-up (years) & $12 \pm 7$ & $7 \pm 7$ & $10 \pm 7$ & $9 \pm 7$ & $10 \pm 7$ & $11 \pm 7$ & $<0.001$ \\
\hline
\end{tabular}

\footnotetext{
$\mathrm{N}=1368$ unless otherwise specified.
No statistical pair comparisons were completed because of the number of groups.

No statistical pair comparisons were completed because of the number of $g$ rou
* Value for the comparison across the six groups after adjustment for age.
}

group was associated with an increased all-cause mortality risk, whereas subjects with inconsistent obstructive pattern were not at increased risk for mortality. The mixed pattern was also associated with a significant adjusted HR for all-cause mortality of 1.4 (95\% CI 1.1 to 1.8). In figure 1, survival curves adjusted for sex, age and BMI are shown for the six longitudinal spirometric patterns. Unadjusted survival curves are shown in figure E1 in the online supplement. Because subjects had to complete at least one follow-up survey to be eligible for longitudinal analyses, sensitivity analyses were completed after defining survey 2 as the first time point in survival models. The results were unchanged.

Similarly, when all-cause mortality risks were compared across the three cross-sectional spirometric patterns at enrolment, both the restrictive and the obstructive spirometric patterns at enrolment were associated with sex-, age- and BMIadjusted increased mortality risk compared with subjects with normal lung function (adjusted HR 1.4 (95\% CI 1.2 to 1.7) and 1.9 (95\% CI 1.6 to 2.2 ), respectively).

Profiles of specific causes of death differed substantially across longitudinal spirometric groups. Subjects with the recurrent obstructive pattern were at increased risk of dying of heart disease, lung cancer, COPD, stroke and diabetes. In particular, as expected, their risk of dying from COPD was very high (adjusted HR 38.0 (95\% CI 15.6 to 92.6). The risk of dying from COPD was also significantly increased among subjects with the mixed longitudinal pattern, but not among subjects with either the recurrent or inconsistent restrictive pattern. Subjects in either the recurrent or inconsistent restrictive pattern groups had the highest risk for dying of heart disease and diabetes.

\section{DISCUSSION}

In this study of the long-term population-based TESAOD cohort we found that (1) $12 \%$ of the population had a restrictive spirometric pattern at enrolment; (2) this restrictive spirometric pattern was associated with respiratory symptoms but not with smoking, serum IgE, allergy skin tests or eosinophilia; (3) in longitudinal analyses, $38 \%$ of subjects with the restrictive pattern at enrolment developed airway obstruction during the follow-up; (4) the remaining $62 \%$ had either a recurrent or inconsistent chronic restrictive spirometric pattern and were at significantly increased risk of dying from all causes, including a high risk of dying from heart disease and dying from or with diabetes.

Our finding that $12 \%$ of the TESAOD cohort had a restrictive spirometric pattern at the baseline survey is comparable with cross-sectional data reported by Mannino and colleagues from the NHANES I (9\%), ${ }^{8}$ the Cardiovascular Health Study $(10 \%)^{10}$ and the ARIC $(13 \%)^{9}$ adult cohorts. However, when the TESAOD subjects were re-evaluated during the follow-up, only one-third of them showed a recurrent restrictive pattern while $29 \%$ had an inconsistent restrictive pattern and 38\% developed an obstructive pattern. The latter group was associated with both a positive smoking history and an asthma diagnosis at enrolment (data not shown).

These findings suggest that, although in about one-third of cases a restrictive pattern on a single spirometric test may convert into an obstructive pattern over time, in the majority of cases the restrictive spirometric pattern is not due to an underlying obstructive lung disease. Consistent with this scenario, subjects in the restrictive group had smoking rates comparable

Table 3 Sex-, age- and BMI-adjusted mortality hazard ratios and corresponding 95\% Cls associated with the six longitudinal spirometric patterns

\begin{tabular}{|c|c|c|c|c|c|c|c|}
\hline \multirow[b]{2}{*}{ Longitudinal spirometric patterns } & \multicolumn{7}{|c|}{ Underlying cause of death } \\
\hline & All-cause & Heart disease & Cancer & Lung cancer & COPD & Stroke & Diabetes \\
\hline Consistent normal $(\mathrm{N}=883$ ) & Reference & Reference & Reference & Reference & Reference & Reference & Reference \\
\hline Inconsistent restrictive $(\mathrm{N}=60)$ & $1.9(1.4$ to 2.6$)$ & 2.7 (1.7 to 4.3$)$ & $1.4(0.7$ to 3.0$)$ & $1.5(0.4$ to 6.6$)$ & No events & 3.5 (1.2 to 9.8$)$ & $6.0(1.9$ to 19.2$)$ \\
\hline Recurrent obstructive ( $N=153$ ) & 2.4 (1.9 to 2.9$)$ & $2.1(1.4$ to 3.0$)$ & $1.4(0.8$ to 2.2$)$ & $3.2(1.5$ to 6.6$)$ & 38.0 (15.6 to 92.6$)$ & $6.2(2.9$ to 13.4$)$ & $5.4(2.0$ to 15.2$)$ \\
\hline Inconsistent obstructive $(\mathrm{N}=52)$ & 1.2 (0.8 to 1.7$)$ & $1.3(0.8$ to 2.3$)$ & $0.7(0.3$ to 1.8$)$ & No events & $4.2(0.8$ to 21.1$)$ & $1.5(0.3$ to 6.5$)$ & $1.0(0.1$ to 7.7$)$ \\
\hline
\end{tabular}




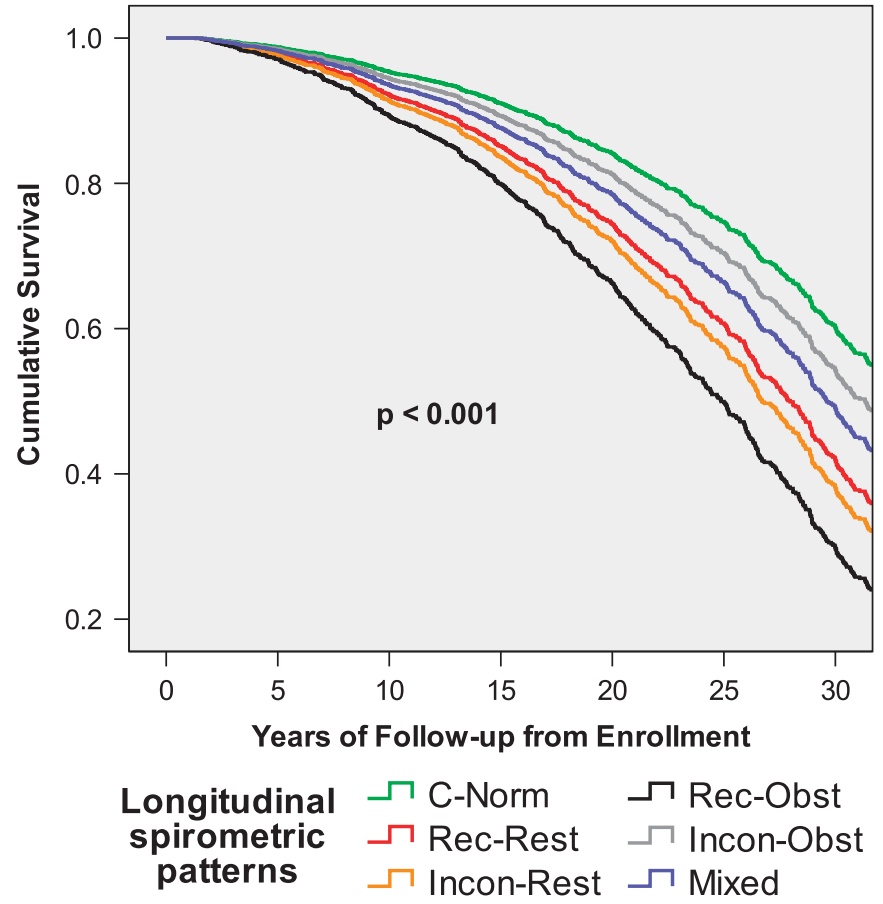

Figure 1 Survival curves adjusted for sex, age and body mass index associated with the six longitudinal spirometric patterns. C-Norm, consistent normal; Rec-Rest, recurrent restrictive; Incon-Rest, inconsistent restrictive; Rec-Obst, recurrent obstructive; Incon-Obst, inconsistent obstructive; Mixed, mixed restrictive/obstructive.

to those of participants in the normal group and significantly lower than those of subjects in the obstructive group, indicating that they did not have a form of cigarette-related COPD. They also had less asthma and lower IgE levels than did subjects in the obstructive group, which also argues against a link between the restrictive spirometric pattern and latent airway obstruction. Indeed, although subjects who had a restrictive pattern at enrolment had more diagnoses of asthma than subjects in the normal group, this association was mainly due to subjects who developed a subsequent obstructive pattern since only $8 \%$ of subjects with either a recurrent or inconsistent longitudinal restrictive pattern had a physician-confirmed diagnosis of asthma at enrolment (compared with $6.4 \%$ of subjects in the consistent normal group and $33.5 \%$ of subjects with a recurrent obstructive pattern).

Factors that are causally linked to spirometric restriction remain largely unknown. Obesity and heart failure are potential common causes of spirometric restriction. ${ }^{23}{ }^{24}$ However, obesity is unlikely to play a major role in our study because $<15 \%$ of subjects with the restrictive spirometric pattern were obese at enrolment and overweight rates were largely comparable across subjects with the restrictive, obstructive and normal patterns. Data on heart failure were not available in our study, but heart disease was reported at enrolment by $26 \%$ of subjects in the restrictive group, only a minority of whom presumably suffered from heart failure.

In our study, both the recurrent and the inconsistent restrictive spirometric patterns were strongly associated with all-cause mortality. In addition, subjects in either the recurrent or inconsistent restrictive pattern groups had particularly high risks for dying of heart disease and diabetes. These findings suggest that spirometric restriction accounts for a substantial proportion of the association between lung function impairment and these comorbidities, although the nature of these links remains to be determined. The reasons why subjects with an inconsistent restrictive pattern had a mortality risk largely comparable to that of subjects with a recurrent restrictive pattern, despite the greater lung function impairment of the latter, also remain to be determined. However, it is noteworthy that the recurrent and inconsistent restrictive groups included a similar number of subjects, whereas the recurrent to inconsistent obstructive pattern ratio was almost $3: 1$, suggesting that spirometric restriction may be more likely than obstruction to resolve and relapse inconsistently over time. Taken together, these observations indicate the importance of an early identification of subjects who present with a restrictive spirometric pattern, a phenotype whose sequelae are frequently underestimated in the clinical setting.

A limitation of our study is that, like most other large epidemiological cohorts, it lacks data on lung function after bronchodilator. Thus, we could not determine in what proportion of subjects in the restrictive and obstructive groups the abnormal spirometric pattern was reversible (ie, it would resolve into a normal pattern following administration of a bronchodilator). Also, the possibility that clinical conditions such as heart failure may have developed in some participants over time and, in turn, influenced the likelihood of these subjects to be included in one of the restrictive longitudinal patterns cannot be ruled out. However, this potential limitation would apply to the longitudinal spirometric patterns and, thus, it could not explain the strong link between the restrictive pattern assessed at enrolment and subsequent mortality risk. Finally, differences in the length of follow-up may influence the likelihood of participants to have a recurrent versus inconsistent longitudinal pattern because subjects with longer follow-up have more chances to alternate normal and abnormal lung function tests during the study. To minimise the impact of such a potential artefact, we defined inconsistent and recurrent patterns based on the proportion of follow-up surveys with normal or abnormal lung function tests, a parameter that should be relatively independent of the length of follow-up. Subjects with recurrent restrictive or obstructive longitudinal spirometric patterns had a shorter mean length of follow-up than subjects with a consistently normal pattern (table 2). However, these differences may be due to the different mortality risk among these groups.

The strengths of our study are the population-based prospective nature of the TESAOD cohort, the extensive phenotypic data and long-term follow-up period, and the exhaustive search for information on vital status of participants.

In summary, our findings indicate that a restrictive spirometric pattern is present in a substantial proportion of the general adult population and that, in most cases, it is not associated with the subsequent development of an obstructive pattern. Subjects with the restrictive pattern show a profile of clinical characteristics and risk factors largely distinct from those of subjects with obstructive lung disease. Most importantly, they carry a significant burden of respiratory symptoms, functional impairment and risk of mortality by comorbidities, even among those with an inconsistent restrictive pattern. Clinical evaluations of potential interventions to reduce the morbidity and mortality burden associated with spirometric restriction are needed.

Funding This study was funded by grants HL14136, HL085195 and HL095021 from the National Heart, Lung and Blood Institute, a grant award by the American Thoracic Society/Alpha1 Foundation, grant $0660059 Z$ by the American Heart Association and an unrestricted grant from the Barry and Janet Lang Donor Advised Fund. SG was the recipient of a Parker B. Francis Fellowship.

Competing interests None 
Ethics approval This study was conducted with the approval of the Institutional Review Board of the University of Arizona.

Provenance and peer review Not commissioned; externally peer reviewed.

\section{REFERENCES}

1. Speizer FE, Fay ME, Dockery DW, et al. Chronic obstructive pulmonary disease mortality in six U.S. cities. Am Rev Respir Dis 1989;140(3 Pt 2):S49-55.

2. Hole DJ, Watt GC, Davey-Smith G, et al. Impaired lung function and mortality risk in men and women: findings from the Renfrew and Paisley prospective population study. BMJ 1996:313:711-15;discussion 715-16.

3. Pauwels RA, Buist AS, Calverley PM, et al. Global strategy for the diagnosis, management, and prevention of chronic obstructive pulmonary disease. NHLBI/WHO Global Initiative for Chronic Obstructive Lung Disease (GOLD) Workshop summary. Am J Respir Crit Care Med 2001;163:1256-76

4. Jemal A, Ward E, Hao $Y$, et al. Trends in the leading causes of death in the United States, 1970-2002. JAMA 2005;294:1255-9.

5. Mathers CD, Loncar D. Projections of global mortality and burden of disease from 2002 to 2030. PLoS Med 2006;3:e442.

6. Ashley F, Kannel WB, Sorlie PD, et al. Pulmonary function: relation to aging, cigarette habit, and mortality. Ann Intern Med 1975;82:739-45

7. Mannino DM, Ford ES, Redd SC. Obstructive and restrictive lung disease and functional limitation: data from the Third National Health and Nutrition Examination. J Intern Med 2003:254:540-7.

8. Mannino DM, Buist AS, Petty TL, et al. Lung function and mortality in the United States: data from the First National Health and Nutrition Examination Survey follow up study. Thorax 2003:58:388-93.

9. Mannino DM, Doherty DE, Sonia Buist A. Global Initiative on Obstructive Lung Disease (GOLD) classification of lung disease and mortality: findings from the Atherosclerosis Risk in Communities (ARIC) study. Respir Med 2006;100:115-22.

10. Mannino DM, Davis KJ. Lung function decline and outcomes in an elderly population. Thorax 2006;61:472-7.

11. Ford ES, Mannino DM. Prospective association between lung function and the incidence of diabetes: findings from the National Health and Nutrition Examination Survey Epidemiologic Follow-up Study. Diabetes Care 2004:27:2966-70.
12. Mannino DM, Thorn D, Swensen A et al. Prevalence and outcomes of diabetes, hypertension and cardiovascular disease in COPD. Eur Respir J 2008;32:962-9.

13. Leone N, Courbon D, Thomas F, et al. Lung function impairment and metabolic syndrome: the critical role of abdominal obesity. Am J Respir Crit Care Med 2009:179:509-16.

14. Fimognari FL, Pasqualetti $P$, Moro $L$, et al. The association between metabolic syndrome and restrictive ventilatory dysfunction in older persons. J Gerontol A Biol Sci Med Sci 2007;62:760-5.

15. Mannino DM, Holguin F, Pavlin Bl, et al. Risk factors for prevalence of and mortality related to restriction on spirometry: findings from the First National Health and Nutrition Examination Survey and follow-up. Int J Tuberc Lung Dis 2005;9:613-21.

16. Johnston AK, Mannino DM, Hagan GW, et al. Relationship between lung function impairment and incidence or recurrence of cardiovascular events in a middle-aged cohort. Thorax 2008;63:599-605

17. Lebowitz MD, Knudson RJ, Burrows B. Tucson epidemiologic study of obstructive lung diseases. I: Methodology and prevalence of disease. Am J Epidemiol 1975; 102:137-52.

18. Knudson RJ, Slatin RC, Lebowitz MD, et al. The maximal expiratory flow-volume curve. Normal standards, variability, and effects of age. Am Rev Respir Dis 1976:113:587-600.

19. Knudson RJ, Lebowitz MD, Holberg CJ, et al. Changes in the normal maximal expiratory flow-volume curve with growth and aging. Am Rev Respir Dis 1983; 127:725-34.

20. Burrows B, Martinez FD, Halonen M, et al. Association of asthma with serum lgE levels and skin-test reactivity to allergens. N Engl J Med 1989;320:271-7.

21. Schisterman EF, Whitcomb BW. Use of the Social Security Administration Death Master File for ascertainment of mortality status. Popul Health Metr 2004;2:2.

22. Davis KB, Fisher L, Gillespie MJ, et al. A test of the National Death Index using the Coronary Artery Surgery Study (CASS). Control Clin Trials 1985;6:179-91.

23. Ray CS, Sue DY, Bray G, et al. Effects of obesity on respiratory function. Am Rev Respir Dis 1983:128:501-6.

24. Faggiano P. Abnormalities of pulmonary function in congestive heart failure. Int $J$ Cardiol 1994:44:1-8. 